International Journal of Pure and Applied Mathematics

Volume 99 No. 3 2015, 299-313

ISSN: 1311-8080 (printed version); ISSN: 1314-3395 (on-line version)

url: http://www.ijpam.eu

doi: http://dx.doi.org/10.12732/ijpam.v99i3.6

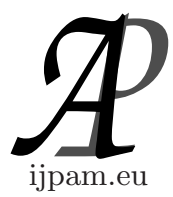

\title{
TRUST REGION WITH NONLINEAR CONJUGATE GRADIENT METHOD
}

\author{
Dalal Hachelfi ${ }^{1}$, Yamina Laskri ${ }^{2}$, Mohamed Lamine Sahari ${ }^{3}$ \\ ${ }^{1,2,3}$ Department of Mathematics \\ Badji Mokhtar University \\ P.O. Box 12, Annaba, 23000, ALGERIA \\ ${ }^{1}$ dalelhach@yahoo.fr
}

Abstract: Descent direction methods and trust region methods are usually used to solve the unconstrained optimization problem $(p)$

$$
(P)\left\{\min _{x \in \mathbb{R}^{n}} f(x) .\right.
$$

In this work, we are interested in convergence results that use trust region methods which employ the conjugate gradient method Day-Yuan version as a subprogram for each iteration. Further, we penalize the quadratic problems with constraints and convert them into series of unconstrained problems.

AMS Subject Classification: 65K05, 90C30

Key Words: unconstrained optimization, conjugate gradient method DayYuan version, Wolf's rule, trust region methods, penalty method

\section{Introduction}

The aim of this paper is to solve the following unconstrained optimization problem

$$
(P):\left\{\min _{x \in \mathbb{R}^{n}} f(x),\right.
$$

where the function $f$ is assumed to be nonlinear and differentiable.

Received: October 29, 2014

(c) 2015 Academic Publications, Ltd. url: www.acadpubl.eu 
At the current point $x_{k} \in \mathbb{R}^{n}$, a model of the variation of $f$, for an increment $d$ of $x_{k}$, is supposed to be given. In differentiable optimization, it is reasonable to consider a quadratic model of the form

$$
f\left(x_{k}+d\right) \simeq \Psi_{k}(d) \simeq f\left(x_{k}\right)+g_{k}^{T} d+\frac{1}{2} d^{T} H_{k} d
$$

where $g_{k}=\nabla f\left(x_{k}\right)$ is the gradient of $f$ at $x_{k}$ and $H_{k}=\nabla^{2} f\left(x_{k}\right)$ is the Hessian of $f$ at $x_{k} . g_{k}$ and $H_{k}$ are assumed to be computed using the Euclidean scalar product $\langle u, v\rangle=\sum_{i} u_{i} v_{i}$.

In trust region methods $[1,2,3,7]$, we consider that $\Psi_{k}$ is a model of the variation of $f$ which is acceptable in a neighborhood of the form

$$
\bar{B}\left(0, \triangle_{k}\right)=\left\{x \in \mathbb{R}^{n}:\|x\| \leq \triangle_{k}\right\}
$$

where $\triangle_{k}>0$ and $\|$.$\| is the Euclidean norm. The domain \bar{B}\left(0, \triangle_{k}\right)$ is called the Trust region of the model $\Psi_{k}$ and the positive number $\triangle_{k}$ is called the radius of trust.

To find the increase $d_{k}$ to be given to $x_{k}$, we minimize the quadratic model $\Psi_{k}$ on the trust region [7]. Therefore, we have to solve the quadratic subproblem

$$
\left(p_{1}\right)\left\{\begin{array}{l}
\min \Psi_{k}(d) \\
\|d\| \leq \triangle_{k}
\end{array}\right.
$$

A possible approach for solving constraint problems is the penalty method [7]. Clearly, we introduce the constraints in the objective under penalties form by starting from a non-feasible solution and try to impose on the unconstrained optimum to arrive to qualifying set.

By solving a problem of the form

$$
\left(p_{2}\right)\left\{\min _{d \in \mathbb{R}^{n}} \Phi\left(d, \mu_{k}\right)=\min _{d \in \mathbb{R}^{n}}\left\{\Psi(d)+\mu_{k} p(d)\right\},\right.
$$

the sequence of values $\left\{\mu_{k}\right\}$ has the following properties

i) $\mu_{k}>0, \forall k$,

ii) the sequence $\left\{\mu_{k}\right\}$ is increasing. That is $\mu_{k+1}>\mu_{k}$ for every $k$,

iii) the sequence $\left\{\mu_{k}\right\}$ diverges as $k \rightarrow \infty$, 
and the $p(d)$ is a continuous penalty function such that

$$
p(d)=\left\{\begin{array}{cc}
=0 & \text { if } \quad\|d\| \leq \triangle \\
>0 & \text { otherwise }
\end{array}\right.
$$

We choose

$$
p(d)=(\max \{0,\|d\|-\triangle\})^{2}
$$

\section{Algorithm 1.}

Initialization step. Set constants $\eta_{1}, \eta_{2}, \gamma_{1}, \gamma_{2}$ and $\gamma_{3}$ such as

$$
0<\eta_{1}<\eta_{2}<1 \text { et } 0<\gamma_{1}<\gamma_{2}<1 \leq \gamma_{3} .
$$

Set the trust radius $\Delta_{0}$ and an iterate $x_{0}$. Compute of $f\left(x_{0}\right)$. Put $k=0$ and go to step 1.

Step 1. Construct the model function $\Phi\left(d, \mu_{k}\right)$ according to the relation (1.4) and determine an approximate solution $d_{k}$ of the problem $\left(p_{2}\right)$

$$
\left(p_{2}\right)\left\{\min _{d \in \mathbb{R}^{n}} \Phi\left(d, \mu_{k}\right) .\right.
$$

Step 1.0. Set a penalized problem $\phi_{k}\left(d, \mu_{k}\right)$ and $\mu_{0}>0, \beta>1$, the start point $s_{0}, M_{0}=\nabla \phi\left(d_{0}, \mu_{0}\right)$, put $s_{0}=-M_{0}$. Set $k=0$ and go to step 1.1.

Step 1.1. If $M_{k}=0:$ stop $\left(d^{*}=d_{k}\right)$. "Stop test".

Otherwise go to step 1.2.

Step 1.2. Put $\mu_{k+1}=\beta \mu_{k}$.

Define $d_{k+1}=d_{k}+\alpha_{k} s_{k}$ with $\alpha_{k}$ satisfies the conditions of low Wolfe

$$
\left\{\begin{array}{c}
\phi\left(d_{k}+\alpha_{k} s_{k}, \mu_{k}\right) \leq \phi\left(d_{k}, \mu_{k}\right)+\rho \alpha s_{k}^{T} M_{k} \text { such that } 0<\rho<\sigma<1 . \\
s_{k}^{T} M_{k+1} \geq \sigma s_{k}^{T} M_{k} \\
s_{k}=-M_{k+1}+B_{k+1} s_{k}
\end{array}\right.
$$




$$
B_{k+1}^{D Y}=\frac{\left\|M_{k+1}\right\|^{2}}{s_{k}^{T}\left[M_{k+1}-M_{k}\right]}=\frac{\left\|M_{k+1}\right\|^{2}}{s_{k}^{T} y_{k}} .
$$

Set $k=k+1$ and go to step 1.1.

Step 2. Cmpute of $f\left(x_{k}+d_{k}\right)$ and evaluate the performance criteria

$$
\rho_{k}=\frac{f\left(x_{k}\right)-f\left(x_{k}+d_{k}\right)}{\Psi\left(x_{k}\right)-\Psi\left(x_{k}+d_{k}\right)} .
$$

Step 3. Update the radius of trust

(a) If $\rho_{k} \geq \eta_{2}$ : The step is a success and $d_{k}$ is accepted. $x_{k+1}=x_{k}+d_{k}$ and choose $\triangle_{k} \in\left[\triangle_{k}, \gamma_{3} \triangle_{k}\right]$.

(b) If $\eta_{1} \leq \rho_{k} \leq \eta_{2}$ : The step is a success and $d_{k}$ is accepted. $x_{k+1}=x_{k}+d_{k}$ and choose $\triangle_{k} \in\left[\gamma_{2} \triangle_{k}, \triangle_{k}\right]$.

(c) If $\rho_{k}<\eta_{1}$ : The step is a failure and $d_{k}$ is rejected. $x_{k+1}=x_{k}$ and choose $\triangle_{k} \in\left[\gamma_{1} \triangle_{k}, \gamma_{2} \triangle_{k}\right]$.

Step 4. If $\left\|\nabla f\left(x_{k}\right)\right\| \leq \varepsilon$, then the algorithm is stopped. Otherwise, we set $k=k+1$ and go back to step 1 .

We usually choose $\varepsilon=10^{-5}, \eta_{1}=0,25, \eta_{2}=0,75, \gamma_{1}=0,25, \gamma_{2}=0,75$ and $\gamma_{3}=2$.

\section{Convergence Results}

By a similar manner to the linear search methods, the determination of optimal steps $d_{k}$ is not a necessary condition for global convergence. Under certain conditions, a good approximation of these steps may be acceptable. It suffices to determine an approximate solution $d_{k}$, in the interior of the trust region, which produces a sufficient reduction of the model function. This reduction can be achieved by the method of Cauchy point $d_{k}^{c}$.

Definition 1. [3] We call Cauchy point of quadratic subproblem $\left(R C_{k}\right)$, the point noted $d_{k}^{c}$ solution of the problem

$$
\left(p_{3}\right)\left\{\begin{array}{l}
\min \Psi_{k}(d) \\
\|d\| \leq \triangle_{k} \\
d=-\alpha g_{k}, \alpha \in \mathbb{R} .
\end{array}\right.
$$


Therefore, such a point is the point minimizing $\Psi_{k}$ in the trust region. That is, along the right side of the strongest slope of $\Psi_{k}$.

Proposition 2. [3] The Cauchy point $d_{k}^{c}$ is unique and it is given by

$$
d_{k}^{c}= \begin{cases}0 & \text { if } g_{k}=0 \\ -\frac{\triangle_{k}}{\left\|g_{k}\right\|} g_{k} & \text { if } g_{k} \neq 0 \text { et } g_{k}^{T} H_{k} g_{k} \leq 0 \\ -\frac{\triangle_{k}}{\left\|g_{k}\right\|} g_{k} \min \left(\frac{\left\|g_{k}\right\|^{3}}{\triangle_{k} g_{k}^{T} H_{k} g_{k}}, 1\right) & \text { otherwise. }\end{cases}
$$

Proof. Indeed, the case where $g_{k}=0$ is obvious. Suppose now that $g_{k} \neq 0$ . If $g_{k}^{T} H_{k} g_{k} \leq 0$, then $\alpha \mapsto \Psi_{k}\left(-\alpha g_{k}\right)$ is concave and $\nabla \Psi_{k}(0)^{T}\left(-g_{k}\right)=$ - $\left\|g_{k}\right\|^{2}<0$, therefore, $\alpha$ must be taken as large as possible while keeping $\left\|-\alpha g_{k}\right\| \leq \triangle_{k}$. This gives $\alpha=\frac{\triangle_{k}}{\left\|g_{k}\right\|}$.

It remains to examine the case $g_{k}^{T} H_{k} g_{k}>0$. The result follows from the fact that, in this case, the minimum of the function $\alpha \mapsto \Psi_{k}\left(-\alpha g_{k}\right)$ is attained for $\alpha=\frac{\left\|g_{k}\right\|^{2}}{g_{k}^{T} H_{k} g_{k}}$.

As we have seen, the point of Cauchy $d_{k}^{c}$ allows whether an approximate solution of the problem in trust region to be validated or not. So, the point $d_{k}$ must verify the decrease of the model function

$$
\Psi_{k}(0)-\Psi_{k}\left(d_{k}\right) \geq c_{1}\left(\Psi_{k}(0)-\Psi_{k}\left(d_{k}^{c}\right)\right) . \quad c_{1}>0
$$

The following proposition is known as Powell Condition.

Proposition 3. [1] The Cauchy point $d_{k}^{c}$ verifies the condition of Powell with $C=\frac{1}{2}$, such as

$$
\Psi_{k}(0)-\Psi_{k}\left(d_{k}^{c}\right) \geq C\left\|g_{k}\right\| \min \left\{\frac{\left\|g_{k}\right\|}{\left\|H_{k}\right\|}, \triangle_{k}\right\} .
$$

Proof. We consider two cases for the Hessian matrix $H_{k}$. That is,

a) $g_{k}^{T} H_{k} g_{k} \leq 0$

$$
\Psi_{k}\left(d_{k}^{c}\right)-\Psi_{k}(0)=\Psi_{k}\left(-\frac{\triangle_{k}}{\left\|g_{k}\right\|} g_{k}\right)-f_{k}
$$




$$
\begin{aligned}
& =-\frac{\triangle_{k}}{\left\|g_{k}\right\|}\left\|g_{k}\right\|^{2}+\frac{1}{2} \frac{\triangle_{k}^{2}}{\left\|g_{k}\right\|^{2}} g_{k}^{T} H_{k} g_{k} \\
& \leq-\frac{\triangle_{k}}{\left\|g_{k}\right\|}\left\|g_{k}\right\|^{2} \\
& \leq-\triangle_{k}\left\|g_{k}\right\| \\
& \leq-\left\|g_{k}\right\| \min \left\{\frac{\left\|g_{k}\right\|}{\left\|H_{k}\right\|}, \triangle_{k}\right\} \\
& \leq-\frac{1}{2}\left\|g_{k}\right\| \min \left\{\frac{\left\|g_{k}\right\|}{\left\|H_{k}\right\|}, \triangle_{k}\right\}
\end{aligned}
$$

so, we have (3.3).

b) $\mathbf{g}_{k}^{T} \mathbf{H}_{k} \mathbf{g}_{k}>\mathbf{0}$ and $\frac{\left\|g_{k}\right\|^{3}}{\triangle_{k} \mathbf{g}_{k}^{T} \mathbf{H}_{k} \mathbf{g}_{k}} \leq 1$ :

$$
\begin{aligned}
\Psi_{k}\left(d_{k}^{c}\right)-\Psi_{k}(0) & =-\frac{\left\|g_{k}\right\|^{4}}{\triangle_{k} \mathbf{g}_{k}^{T} \mathbf{H}_{k} \mathbf{g}_{k}}+\frac{1}{2} g_{k}^{T} H_{k} g_{k} \frac{\left\|g_{k}\right\|^{4}}{\left(\mathbf{g}_{k}^{T} \mathbf{H}_{k} \mathbf{g}_{k}\right)^{2}} \\
& =-\frac{1}{2} \frac{\left\|g_{k}\right\|^{4}}{\mathbf{g}_{k}^{T} \mathbf{H}_{k} \mathbf{g}_{k}} \\
& \leq-\frac{1}{2} \frac{\left\|g_{k}\right\|^{4}}{\left\|\mathbf{H}_{k}\right\| \mathbf{g}_{k} \|^{2}} \\
& \leq-\frac{1}{2} \frac{\left\|g_{k}\right\|^{2}}{\left\|\mathbf{H}_{k}\right\|} \\
& \leq-\frac{1}{2}\left\|g_{k}\right\| \min \left\{\frac{\left\|g_{k}\right\|}{\left\|H_{k}\right\|}, \triangle_{k}\right\}
\end{aligned}
$$

so,we have (3.3).

c) $\mathbf{g}_{k}^{T} \mathbf{H}_{k} \mathbf{g}_{k}<\frac{\left\|g_{k}\right\|^{3}}{\triangle_{k}}, \quad d_{k}^{c}=-\frac{\triangle_{k}}{\left\|g_{k}\right\|} g_{k}$

we have (3.3) .

Theorem 4. [1] Let $d_{k}$ be an arbitrary vector such that

$$
\left\|d_{k}\right\| \leq \triangle_{k}
$$

and

$$
\Psi_{k}(0)-\Psi_{k}\left(d_{k}\right) \geq c_{2}\left(\Psi_{k}(0)-\Psi_{k}\left(d_{k}^{c}\right)\right) .
$$


Then, $d_{k}$ satisfies (3.3) with $c_{2}=\frac{c_{1}}{2}$.

Proof. Since $\left\|d_{k}\right\| \leq \triangle_{k}$, from (3.2), we have,

$$
\Psi_{k}(0)-\Psi_{k}\left(d_{k}\right) \geq c_{1}\left(\Psi_{k}(0)-\Psi_{k}\left(d_{k}^{c}\right)\right) \geq \frac{1}{2} c_{1}\left\|g_{k}\right\| \min \left\{\frac{\left\|g_{k}\right\|}{\left\|H_{k}\right\|}, \triangle_{k}\right\}
$$

In particular, if $d_{k}$ is the exact solution of $\left(R C_{k}\right)$, then it satisfies (3.3) with $c_{2}=\frac{1}{2}$.

\subsection{Convergence of penalty methods}

The following two lemmas are used to prove the convergence of the method.

By $d_{k}$ we denote the solution of $\left(p_{2}\right)$.

Lemma 5. [7] For any value of $k$, we have

i) $\Phi\left(d_{k}, \mu_{k}\right) \leq \Phi\left(d_{k+1}, \mu_{k+1}\right)$

ii) $p\left(d_{k}\right) \geq p\left(d_{k+1}\right)$

iii) $\Psi\left(d_{k}\right) \leq \Psi\left(d_{k+1}\right)$

\section{Proof.}

i) Since the sequence $\left\{\mu_{k}\right\}$ is increasing and $p\left(d_{k+1}\right) \geq 0$, we have

$$
\begin{aligned}
\Phi\left(d_{k+1}, \mu_{k+1}\right) & =\Psi\left(d_{k+1}\right)+\mu_{k+1} p\left(d_{k+1}\right) \\
& \geq \Psi\left(d_{k+1}\right)+\mu_{k} p\left(d_{k+1}\right) \\
& \geq \Psi\left(d_{k}\right)+\mu_{k} p\left(d_{k}\right)
\end{aligned}
$$

and $d_{k}$ minimizes $\Phi\left(d, \mu_{k}\right)$,we obtain

$$
\Phi\left(d_{k+1}, \mu_{k+1}\right) \geq \Phi\left(d_{k}, \mu_{k}\right) .
$$

ii) Since $d_{k}$ minimizes $\Phi\left(d, \mu_{k}\right)$

$$
\Phi\left(d, \mu_{k}\right) \Psi\left(d_{k}\right)+\mu_{k} p\left(d_{k}\right) \leq \Psi\left(d_{k+1}\right)+\mu_{k} p\left(d_{k+1}\right)
$$

and $d_{k+1}$ minimizes $\Phi\left(d, \mu_{k+1}\right)$

$$
\Psi\left(d_{k+1}\right)+\mu_{k+1} p\left(d_{k+1}\right) \leq \Psi\left(d_{k}\right)+\mu_{k+1} p\left(d_{k}\right),
$$


adding the two inequalities we obtain,

$$
\left(\mu_{k+1}-\mu_{k}\right) p\left(d_{k+1}\right) \leq\left(\mu_{k+1}-\mu_{k}\right) p\left(d_{k}\right)
$$

Since $\mu_{k+1}>\mu_{k}$, we have

$$
p\left(d_{k+1}\right) \leq p\left(d_{k}\right)
$$

iii) $\Psi\left(d_{k+1}\right)+\mu_{k} p\left(d_{k+1}\right) \geq \Psi\left(d_{k}\right)+\mu_{k} p\left(d_{k}\right)$ using ii) and the fact that $\mu_{k} \geq 0$, we have

$$
\Psi\left(d_{k+1}\right)+\mu_{k} p\left(d_{k+1}\right) \geq \Psi\left(d_{k}\right)+\mu_{k} p\left(d_{k+1}\right) .
$$

Consequently, $\Psi\left(d_{k+1}\right) \geq \Psi\left(d_{k}\right)$.

Lemma 6. [7] $d^{*}$ be an optimal solution of $\left(p_{1}\right)$. Then, for each $k$ we have,

$$
\Psi\left(d^{*}\right) \geq \Phi\left(d_{k}, \mu_{k}\right) \geq \Psi\left(d_{k}\right)
$$

Proof.

$$
\begin{aligned}
\Psi\left(d^{*}\right) & =\Psi\left(d^{*}\right)+\mu_{k} p\left(d^{*}\right) \forall k, \text { since } p\left(d^{*}\right)=0 \\
& \geq \Psi\left(d_{k}\right)+\mu_{k} p\left(d_{k}\right)=\Phi\left(d_{k}, \mu_{k}\right) \forall k\left(\text { since } d_{k} \text { minimizes } \Phi\left(d, \mu_{k}\right)\right) \\
& \geq \Psi\left(d_{k}\right) \forall k\left(\text { since } \mu_{k} \geq 0 \text { and } p\left(d_{k}\right) \geq 0\right) .
\end{aligned}
$$

So, $\Psi\left(d^{*}\right) \geq \Phi\left(d_{k}, \mu_{k}\right) \geq \Psi\left(d_{k}\right) \forall k$.

Theorem 7. Suppose that $d_{k}$ is an exact global minimum of $\left(p_{2}\right)$ and assume that $\left\{\mu_{k}\right\}$ is a divergent sequence. Then, every limit point $d^{*}$ of the sequence $\left\{d_{k}\right\}$ is a solution of $\left(p_{1}\right)$.

Proof. Let $\bar{d}$ be the solution of $\left(p_{1}\right)$ such that

$$
\Psi(\bar{d}) \leq \Psi(d) \forall\|d\| \leq \triangle
$$

Since $d_{k}$ minimizes $\Phi\left(d, \mu_{k}\right)$ for all $k$, we have,

$$
\Phi\left(d_{k}, \mu_{k}\right) \leq \Phi\left(\bar{d}, \mu_{k}\right)
$$

So,

$$
\left.\Psi\left(d_{k}\right)+\mu_{k} p\left(d_{k}\right) \leq \Psi(\bar{d})+\mu_{k} p(\bar{d})=\Psi(\bar{d}) \text { (because } p(\bar{d})=0\right)
$$


Then,

$$
p\left(d_{k}\right) \leq \frac{1}{\mu_{k}}\left[\Psi(\bar{d})-\Psi\left(d_{k}\right)\right]
$$

It is assumed that $d^{*}$ is the limit point $\left\{d_{k}\right\}$. Then, there exists an infinite subsequence of $K$ such that

$$
\lim _{k \in K} d_{k}=d^{*} .
$$

By taking the limit of inequality (3.5), we find

$$
p\left(d^{*}\right)=\lim _{k \in K} p\left(d_{k}\right) \leq \lim _{k \in K} \frac{1}{\mu_{k}}\left[\Psi(\bar{d})-\Psi\left(d_{k}\right)\right]=0
$$

(because $\left\{\mu_{k}\right\}$ is a divergent sequence).

So, we have $p\left(d^{*}\right)=0$ and consequently $\left\|d^{*}\right\| \leq \triangle$.

The optimality of $d^{*}$ follows directly from lemma 6 . Indeed, the relation $\Psi\left(d_{k}\right) \leq \Psi\left(d^{*}\right)$ being satisfied for any $k$, it follows that

$$
\Psi\left(d^{*}\right)=\lim _{k \in K} \Psi\left(d_{k}\right) \leq \Psi(\bar{d}) .
$$

\subsection{Global Convergence}

Theorem 8. Let $f: \mathbb{R}^{n} \rightarrow \mathbb{R}$ be a continuously differentiable function on the set

$$
X=\left\{x \in \mathbb{R}^{n}: f(x)<f\left(x_{0}\right)\right\},
$$

and let $\left\{x_{k}\right\}$ be a sequence generated by the algorithm 1, with $H_{k}$ uniformly bounded. If $d_{k}$ satisfies the condition (3.2) then we have

$$
\liminf _{k \rightarrow \infty}\left\|\nabla f_{k}\right\|=0
$$

Proof. Assume that there exists an indice $k_{1}$ and a constant $\gamma>0$ such that

$$
\left\|\nabla f_{k}\right\| \geq \gamma, \forall k \geq k_{1}
$$

We claim that

$$
\triangle_{k} \rightarrow 0 \quad \text { and } \quad \sum_{k \geq 1}\left\|d_{k}\right\|<\infty .
$$


According to the condition of Powell (3.3) and the fact that $\left\{H_{k}\right\}$ is bounded [3], we have

$$
\begin{aligned}
\Psi_{k}\left(d_{k}\right) & \leq-C\left\|\nabla f_{k}\right\| \min \left\{\frac{\left\|\nabla f_{k}\right\|}{\left\|H_{k}\right\|}, \triangle_{k}\right\} \\
& \leq-C \min \left\{1, \triangle_{k}\right\}
\end{aligned}
$$

where $C$ is a positive constant which is independent of $k$. Using the step 3 of the algorithm $1\left(\rho_{k} \geq \eta_{2}\right)$, we have

$$
f\left(x_{k}+d_{k}\right) \leq f\left(x_{k}\right)+\eta_{2} \Psi_{k}\left(d_{k}\right) .
$$

The last inequality can be written as

$$
\begin{aligned}
& f\left(x_{k+1}\right) \leq f\left(x_{k}\right)-C \min \left\{1, \triangle_{k}\right\}, \\
& C \min \left\{1, \triangle_{k}\right\} \leq f\left(x_{k}\right)-f\left(x_{k+1}\right) .
\end{aligned}
$$

Since $f$ is bounded below, the sequence $\left\{f\left(x_{k}\right)-f\left(x_{k+1}\right)\right\}$ is convergent and we deduce from the above inequality that $\sum_{k \geq 1} \triangle_{k}<\infty$, and since $\left\|d_{k}\right\| \leq$ $\triangle_{k}$, the assertions of (3.8) are deduced.

We now show that the ratio $\rho_{k} \rightarrow 1$. Since $f$ is bounded and $\left\|d_{k}\right\| \leq \triangle_{k}$ then,

$$
\frac{\left|f\left(x_{k}+d_{k}\right)-f\left(x_{k}\right)-\nabla f\left(x_{k}\right)_{k}^{T} d_{k}\right|}{\left\|d_{k}\right\|} \rightarrow 0 .
$$

On the other hand, since $\left\{H_{k}\right\}$ is bounded, we have:

$$
\left|\Psi_{k}\left(d_{k}\right)-\nabla f\left(x_{k}\right)_{k}^{T} d_{k}\right| \leq c\left\|d_{k}\right\|^{2} .
$$

Finally, we can successively write

$$
\begin{gathered}
\rho_{k}=\frac{f\left(x_{k+1}\right)-f\left(x_{k}\right)}{\Psi_{k}\left(d_{k}\right)}=\frac{f\left(x_{k}+d_{k}\right)-f\left(x_{k}\right)-\nabla f\left(x_{k}\right)_{k}^{T} d_{k}}{\Psi_{k}\left(d_{k}\right)} \\
+\frac{\nabla f\left(x_{k}\right)_{k}^{T} d_{k}-\Psi_{k}\left(d_{k}\right)}{\Psi_{k}\left(d_{k}\right)}+1 \\
\left|\rho_{k}-1\right| \leq \frac{\left|f\left(x_{k}+d_{k}\right)-f\left(x_{k}\right)-\nabla f\left(x_{k}\right)_{k}^{T} d_{k}\right|}{\left|\Psi_{k}\left(d_{k}\right)\right|}
\end{gathered}
$$




$$
+\frac{\left|\nabla f\left(x_{k}\right)_{k}^{T} d_{k}-\Psi_{k}\left(d_{k}\right)\right|}{\left|\Psi_{k}\left(d_{k}\right)\right|} .
$$

From (3.9), $\triangle \rightarrow 0$ and $\left\|s_{k}\right\| \leq \triangle_{k}$, and so,

$$
\left|\Psi_{k}\left(d_{k}\right)\right| \geq c\left\|d_{k}\right\| .
$$

Then, combining (3.9) and (3.10), we get

$$
\left|\rho_{k}-1\right| \leq \frac{c\left\|d_{k}\right\|^{2}}{c\left\|d_{k}\right\|} \leq\left\|d_{k}\right\|
$$

This shows that $\rho_{k} \rightarrow 1$.

Accordingly, $\rho_{k}>\eta_{1}$ for $k$ large enough . Due to the updating rule of $\triangle_{k}$ (see the step 3 of the algorithm 1), this implies that $\triangle_{k}>\triangle>0$. But this contradicts the first assertion of (3.8). This contradiction completes the proof.

Theorem 9. Suppose, in addition to the assumptions and conditions of Theorem 8, that $\nabla f_{k}$ is continuous in the sense of Lipchitz and bounded below on the set

$$
X=\left\{x \in \mathbb{R}^{n}: f(x)<f\left(x_{0}\right)\right\},
$$

then we have

$$
\lim _{k \rightarrow \infty}\left\|\nabla f_{k}\right\|=0
$$

Proof. Suppose that there exists a constant $\varepsilon>0$ and a subsequence $\left\{x_{k}\right\}_{k \geq m} \subset B\left(x_{m}, R\right)$ where $B\left(x_{m}, R\right)$ is the closed ball of center $x_{m}$ and radius $R$, for which we have

$$
\left\|\nabla f_{k}\right\| \geq \varepsilon, \quad \text { for all } \quad k \geq m .
$$

Let $l \geq m$ such that $x_{k+1}$ is the first iterate after $x_{m}$ outside $B\left(x_{m}, R\right)$. Since $\left\|\nabla f_{k}\right\| \geq \varepsilon$ for all $k=m, m+1, . ., l$, we can write

$$
f\left(x_{m}\right)-f\left(x_{l+1}\right)=\sum_{k=m}^{l} f\left(x_{k}\right)-f\left(x_{k+1}\right) .
$$

Using the condition of Powell (3.3) and the fact that $\left\{H_{k}\right\}$ is bounded [3], we have as in the proof of Theorem 8 , 


$$
f\left(x_{k+1)}-f\left(x_{k}\right) \leq-C \min \left\{\left\|\nabla f_{k}\right\|, \triangle_{k}\right\} .\right.
$$

So, for all $k \geq m$, we have

$$
f\left(x_{l+1}\right)-f\left(x_{m}\right) \leq-C \min \left\{1, \triangle_{k}\right\} .
$$

Sinse $f\left(x_{l+1}\right)-f\left(x_{m}\right) \rightarrow 0, \min \left\{1, \triangle_{k}\right\}=\triangle_{k}$, for $k$ large enough [3]. Finally, using the fact that $\left\|d_{k}\right\| \leq \triangle_{k}$, we have

$$
\begin{gathered}
\left\|d_{k}\right\| \leq C\left(f\left(x_{k}\right)-f\left(x_{l+1}\right)\right), \quad k=m, m+1, \ldots \ldots . . . \\
\left\|x_{l+1}-x_{m}\right\| \leq \sum_{k=m}^{l}\left\|d_{k}\right\| \leq C\left(f\left(x_{m}\right)-f\left(x_{l+1}\right)\right) .
\end{gathered}
$$

So $\left\|x_{l+1}-x_{m}\right\| \rightarrow 0$. Then by the uniform continuity of $\nabla f_{k}$, we have

$$
\left\|f\left(x_{l+1}\right)-f\left(x_{m}\right)\right\| \rightarrow 0 .
$$

This is in contradiction with (3.12). Hence the theorem is proved.

\section{Numerical tests}

We have performed numerical tests using the trust region algorithm penalized by the following functions

Problem 1: The generalized function of Rosenbrock

$$
f(x)=\sum_{i=1}^{n-1} 100\left(x_{i}^{2}-x_{i+1}\right)^{2}+\left(x_{i}-1\right)^{2}
$$

Problem 2: The generalized function of Dixon

$$
f(x)=\left(x_{1}-1\right)^{2}+\sum_{i=2}^{n} i\left(2 x_{i}^{2}-x_{i-1}\right)^{2}
$$

Problem 3: The function of Oren

$$
f(x)=\left[\sum_{i=1}^{n} i x_{i}^{2}\right]^{2}
$$




\begin{tabular}{|c|c|c|c|c|}
\hline Dimension $n$ & iteration $k$ & $f\left(x_{k}\right)$ & $\left\|g\left(x_{k}\right)\right\|$ & Time of convergence \\
\hline 2 & 26 & $2,59.10^{-12}$ & $9,56.10^{-6}$ & $4,68.10^{-2} \mathrm{~s}$ \\
\hline 10 & 29 & $4,82.10^{-11}$ & $8,71.10^{-6}$ & $0,26 \mathrm{~s}$ \\
\hline 30 & 29 & $8,16.10^{-13}$ & $1,27.10^{-6}$ & $2,51 \mathrm{~s}$ \\
\hline 50 & 28 & $2,72.10^{-11}$ & $7,91.10^{-6}$ & $5,47 \mathrm{~s}$ \\
\hline 80 & 30 & $3.10^{-11}$ & $6,46.10^{-6}$ & $20,57 \mathrm{~s}$ \\
\hline 100 & 28 & $2,71.10^{-11}$ & $8,59.10^{-6}$ & $22 \mathrm{~s}$ \\
\hline 150 & 27 & $5,67.10^{-12}$ & $6,53.10^{-6}$ & $50,66 \mathrm{~s}$ \\
\hline 200 & 25 & $2,11.10^{-13}$ & $5,46.10^{-6}$ & $129,62 \mathrm{~s}$ \\
\hline
\end{tabular}

Table 1: Rosenbrock function

\begin{tabular}{|c|c|c|c|c|}
\hline Dimension $n$ & iteration $k$ & $f\left(x_{k}\right)$ & $\left\|g\left(x_{k}\right)\right\|$ & Time of convergence \\
\hline 2 & 8 & $5,62.10^{-12}$ & $8,72.10^{-6}$ & $1,56.10^{-2} \mathrm{~s}$ \\
\hline 6 & 25 & $1,90.10^{-12}$ & $9,97.10^{-6}$ & $1,06 \mathrm{~s}$ \\
\hline 10 & 41 & 0,66 & $8,43.10^{-6}$ & $1,29 \mathrm{~s}$ \\
\hline 30 & 68 & 0,66 & $9,79.10^{-6}$ & $2,72 \mathrm{~s}$ \\
\hline 50 & 111 & 0,66 & $9,45.10^{-6}$ & $6,83 \mathrm{~s}$ \\
\hline 100 & 36 & 0,66 & $8,84.10^{-6}$ & $39,18 \mathrm{~s}$ \\
\hline
\end{tabular}

Table 2: Dixon function

Problem 4: The function of Powell

$$
\begin{aligned}
f(x)= & \sum_{i=1}^{n / 4}\left[\left(x_{4 i-3}+10 x_{4 i-2}\right)^{2}+5\left(x_{4 i-1}-x_{4 i}\right)^{2}\right. \\
& \left.+\left(x_{4 i-2}-2 x_{4 i-1}\right)^{4}+10\left(x_{4 i-3}-x_{4 i}\right)^{4}\right]
\end{aligned}
$$

In all tests the stopping criterion of the algorithm is $\varepsilon=10^{-5}$. The trust regions algorithm is applied with $\triangle_{0}=0.5, \eta_{1}=0,25, \eta_{2}=0,75, \gamma_{1}=$ $0,25, \gamma_{2}=0,75$ and $\gamma_{3}=2$. The conjugate gradient algorithm is applied to the linear search of wolfe with the parameters: $m_{1}=0.3, m_{2}=0.7$. The penalty method is applied with $\mu_{0}=2$.

The results showing the number of iterations and time of convergence are illustrated in the following tables. 


\begin{tabular}{|c|c|c|c|c|}
\hline Dimension $n$ & iteration $k$ & $f\left(x_{k}\right)$ & $\left\|g\left(x_{k}\right)\right\|$ & Time of convergence \\
\hline 2 & 7 & $2,68.10^{-8}$ & $9,84.10^{-6}$ & $1,56.10^{-2} \mathrm{~s}$ \\
\hline 10 & 19 & $1,10.10^{-8}$ & $9,66.10^{-6}$ & $0,60 \mathrm{~s}$ \\
\hline 50 & 23 & $4,20.10^{-9}$ & $9,74.10^{-6}$ & $103,13 \mathrm{~s}$ \\
\hline 80 & 25 & $3,13.10^{-8}$ & $9,94.10^{-6}$ & $470 \mathrm{~s}$ \\
\hline 100 & 25 & $2,52.10^{-9}$ & $9,32.10^{-6}$ & $967 \mathrm{~s}$ \\
\hline
\end{tabular}

Table 3: Oren Function

\begin{tabular}{|c|c|c|c|c|}
\hline Dimension $n$ & iteration $k$ & $f\left(x_{k}\right)$ & $\left\|g\left(x_{k}\right)\right\|$ & Time of convergence \\
\hline 4 & 20 & $1,15.10^{-8}$ & $9,90.10^{-6}$ & $0,15 \mathrm{~s}$ \\
\hline 8 & 53 & $1,76.10^{-8}$ & $8,89.10^{-6}$ & $0,24 \mathrm{~s}$ \\
\hline 16 & 79 & $9,38.10^{-9}$ & $9,28.10^{-6}$ & $1,04 \mathrm{~s}$ \\
\hline 32 & 18 & $1,25.10^{-8}$ & $4,40.10^{-6}$ & $2,72 \mathrm{~s}$ \\
\hline 100 & 19 & $2,02.10^{-8}$ & $9,30.10^{-6}$ & $31,90 \mathrm{~s}$ \\
\hline 200 & 20 & $3,86.10^{-8}$ & $5,92.10^{-6}$ & $138,37 \mathrm{~s}$ \\
\hline
\end{tabular}

Table 4: Powell function

\section{References}

[1] Andrew R. Conn, Nicholas I. M. Gould and Philippe L. Toint, Trust-Region Methods, MPS-SIAM Series on Optimization, United States (2000).

[2] F. Bastin, V. Malmedy, M. Mouffe, Ph. L. Toint and D. Tomanos, A Retrospective Trust-Region Method for Unconstrained Optimization, Mathematical Programming, 123, No. 2, 395-418.

[3] R. H. Byrd, J-C. Gilbert, and J. Nocedal, A trust region method based on interior point techniques for nonlinear programming, Mathematical Programming, 89 (2000), 149-185, doi: 10.1016/B978-0-12-597050-1.50006-3.

[4] David G. Luenberger, Optimization by Vector Space Methods, Wiley Professional Paperback Series, New York (1969).

[5] Edwin K.P. Chong, Stanislaw H. Zak, An Introduction to Optimization, Wiley-Interscience Series in Discrete Mathematics and Optimization second edition, New York (2001).

[6] J.J. Moré, B.S. Garbow, and K.E. Hillstrom, Testing unconstrained optimization software, ACM Transactions on Mathematical Software, 7, No. 1 (1981), 17-41, ACM0098-3500/81/0300-0017. 
[7] J. Nocedal, S.J. Wright, Numerical Optimization, Springer Series in Operations Research and Financial Engineering, Second Edition, New York (1999).

[8] M.J.D. Powell, A FORTRAN subroutine for unconstrained minimization requiring first derivatives of the objective function, Tech. Rep AERE-R. 6469, Mathematics Branch, A.E.R.E. Harwell, Berkshire, England (1970).

[9] M.J.D. Powell, A new algorithm for unconstrained optimization, In: (Eds: J.B. Rosen, O.L. Mangasarian and K. Ritter), Nonlinear Programming, Academic Press, New York (1970), doi: 10.1007/PL00011391.

[10] M.J.D. Powell, Convergence properties of a class of minimization algorithms, In: (Ed-s: O.L. Mangasarian, R.R. Meyer and S.M. Robinson), Nonlinear Programming, Academic Press, New York (1975), 1-27. 
